\title{
Technical Writer: A proposal to improve quality and documentation in the agile methodology "Scrum"
}

\section{Technical Writer: Una propuesta para mejorar la calidad y la documentación en la metodología ágil "Scrum"}

Kléver Osorio, José Luis Rosero, and Rolando P. Reyes Ch.

Maestría en Ingeniería de Software

Universidad de las Fuerzas Armadas ESPE, Latacunga-Ecuador

\section{Abstract}

Corresponding Author:

Kléver Osorio

Received: 24 December 2019

Accepted: 2 January 2020

Published: 8 January 2020

Publishing services provided by Knowledge E

(c) Kléver Osorio et al. This article is distributed under the terms of the Creative Commons Attribution License, which permits unrestricted use and redistribution provided that the original author and source are credited.

Selection and Peer-review under the responsibility of the SIIPRIN-CITEGC Conference Committee.
Software development companies that choose to work with the agile Scrum methodology focus their efforts on frequent product delivery, neglecting the documentation itself of the processes involved in development. Scrum teams are made up of highly trained technicians in whom trust is placed for the development of a software solution. To support the Scrum team in documentation issues, we propose to incorporate a new actor called Technical Writer into the team, who will be in charge from the beginning of carrying the documentation that merits according to the phases of software development, which will contribute to the delivery of a final product with quality and with the respective documentation. We conclude that although the documentation is not the strength of the agile Scrum methodology, it is very necessary to deliver a quality product to its customers.

Resumen. Las empresas de desarrollo de Software que optan por trabajar con la metodología ágil como Scrum, centran sus esfuerzos en la entrega frecuente y priorizada de productos, descuidando en gran medida la documentación que se genera en sí durante los procesos inmersos en el desarrollo de software. Normalmente, los equipos de Scrum están conformados por técnicos altamente capacitados que se orientan completamente al desarrollo de una solución software. Para apoyar al equipo Scrum en temas de documentación y elaboración de artefactos de software, proponemos incorporar dentro del equipo a un nuevo actor que lo hemos denominado: "Technical Writer", quien se encargará de llevar la documentación desde el principio del proceso Scrum y en las actividades donde se amerite en las fases del desarrollo del software. La propuesta apunta a que los técnicos del equipo Scrum aporten de mejor manera en la entrega de un producto final con calidad y con una adecuada documentación. Concluimos que a pesar de que la documentación no es el fuerte de la metodología ágil Scrum, es muy necesaria para entregar un producto de calidad a sus clientes.

Keywords: Agile methodology, Scrum, documentation, ISO 9001

Palabras Claves: Metodología Ágil, Scrum, documentación, ISO 9001. 


\section{Introducción}

En la actualidad las empresas de desarrollo de software asumen cada día nuevos desafíos en su ámbito de construcción de software. Para lo cual, la mayoría de estas empresas se han inclinado por adoptar las metodologías de desarrollo ágil y su "Manifiesto"[5]. Para el efecto, los expertos crearon 4 valores y 12 principios [6] en Scrum, con los cuales se puede adaptar a la metodología sin influir en costos [7], de esta manera se aumenta el valor comercial y entrega continua de productos software [8].

Al respecto, consideramos a los requerimientos, como punto esencial, los cuales son formulados por los clientes con carácter de urgente, mismos que son presentados a la empresa de desarrollado de software para que sean atendidos a la brevedad posible. Esta aparente urgencia hace que la empresa deba adaptarse rápidamente y hacer suyos los requerimientos de sus clientes, con el fin de mantenerse a la vanguardia en la industria del desarrollo de software y poder solventarlas de una manera oportuna. Para llegar a esto, las empresas consideran que no solamente es importante entregar un producto al cliente con las funcionalidades requeridas, sino que la empresa debe asegurarse que el producto que está entregando se encuentre bien documentado y de calidad.

En específico, la metodología Scrum tiene un enfoque ágil con atributos de colaboración, adaptación y evolución [9, 10], lo que le ha permitido a las empresas tener éxito en la mayoría de sus proyectos en los que han adoptado en trabajar con esta metodología.

Es por ello que las empresas de desarrollo de software consideran que la gestión de la calidad se la debe incorporar desde el inicio de los procesos en específico en la toma de requerimientos, los procesos de desarrollo, pruebas y entrega del producto final. Para el efecto, las empresas toman a la norma ISO 9001:2015. Esta normativa exige llevar la documentación de la información de desarrollo y de aquella documentación necesaria para el aseguramiento del Sistema de Gestión de Calidad. Al respecto, las empresas desarrolladoras de software que utilizan la metodología Scrum saben que en ciertas ocasiones la documentación que se genera es insuficiente para satisfacer los requisitos del estándar ISO 9001:2015. Por lo cual, es necesario incorporar documentación adicional basada en estándares para el aseguramiento de la Calidad.

Por ello, nos motivamos a elaborar este artículo con el fin de presentar una propuesta para apoyar en la elaboración de documentos y artefactos con los equipos de Scrum con un nuevo actor denominado: "Technical Writer", quien se encargará desde el 
principio del proceso Scrum, llevar la documentación que amerite de acuerdo a las fases del desarrollo del software. La propuesta apunta a que los técnicos aporten de mejor manera en la entrega de un producto final con calidad sin desviarse en elaborar documentación.

Este artículo está estructurado de la siguiente manera: En la sección II se exponen algunos trabajos relacionados. En la sección III se presenta Scrum, la metodología ágil en donde se especifica las falencias que presenta en lo que a la documentación respecta. En la Sección IV se describe la metodología propuesta con el nuevo actor incorporado al equipo denominado Technical Writer, con el fin de que satisfaga la documentación requerida por la norma ISO 9001:2015. En la Sección V se muestra la metodología y los resultados obtenidos de un estudio empírico para el desarrollo de un producto software. En la Sección VI presentamos nuestras conclusiones y en la Sección VII damos a conocer los trabajos futuros para demostrar que nuestra propuesta proporciona un sistema de Gestión de la calidad apegado a la norma ISO.

\section{Trabajos relacionados}

Mucho se habla hoy en día, acerca de las metodologías ágiles en la industria de desarrollo de software. Sin embargo, no se habla de que muchos desarrolladores activos sienten una antipatía natural hacia actividades que no están directamente relacionados con escribir código. Visto que hacer otras actividades, desvían a los desarrolladores en codificar una solución software.

Según Christoph Stettina[1] (2011), en su estudio, muestra los resultados de un estudio empírico relacionados a la documentación interna en equipos de desarrollo ágil (Scrum). Este considera que es muy limitada, incluso insuficiente. Por lo que cree que es necesario incorporar documentación apropiada para la transferencia de conocimiento y la mantenibilidad.

Para Michael Coram [2] (2005) indica que según el tipo de proyecto a desarrollar y bajo circunstancias correctas, los proyectos tienen un riesgo bajo con mayor productividad. Sin embargo, un desafío en los métodos ágiles es añadir formalidad en cuanto a documentación para una posible transmisión de conocimientos.

Mientras que Julieta Calabrese [4] en su estudio indica que la metodología Scrum no satisface los requisitos de la documentación que propone la norma ISO 9001:2015. Por lo que diseñaron un único documento incorporando la poca documentación de Scrum, con los estándares que la norma recomienda en las primeras etapas de un proyecto software, para establecer un adecuado sistema de gestión de calidad. 
Esto nos hace pensar que la industria del software durante las últimas décadas ha crecido a un ritmo acelerado. Por lo cual, es necesario la implementación de un Sistema de Gestión de la Calidad de acuerdo a la norma ISO 9001:2015 que apoye a la robustez especialmente de la documentación en el uso de la Metodología Scrum. Es por ello que hemos planteado proponer un nuevo actor al equipo, al que denominaremos "Technical Writer", cuyo rol será determinante en la ejecución del cumplimiento del aseguramiento de la calidad en la entrega de un producto final. La inclusión de un nuevo actor con conocimientos de normas ISO y metodología Scrum permitirá plasmar toda la documentación requerida desde las primeras etapas del proyecto hasta la entrega del producto final, dando un valor agregado a la gestión de la calidad en cuanto a la documentación y se da un primer paso en el camino a la certificación de la empresa en la norma ISO9001:2015.

\section{Scrum}

Scrum [3] es uno de los métodos ágiles más populares no solo en la industria de desarrollo de software, sino también lo es en otras áreas ingenieriles debido a su flexibilidad, lo que permite que esta metodología sea la ideal para sobrellevar los cambios y por sobre todo por la gestión de proyectos. Eso se debe a que Scrum [3] es un Framework iterativo e incremental donde su éxito radica en la autodisciplina y la auto organización del equipo basado en la confianza, inspección y su adaptación a cambios, ofreciendo valor agregado con entregas constantes tan frecuentemente como el cliente lo requiera. Sin embargo, con todo su valor agregado, se deja a un lado la parte formal que es la documentación. Al respecto, El trabajo se organiza en ciclos llamados sprints que son iteraciones de corta duración típicamente de 2 a 4 semanas. Durante cada sprint, el equipo selecciona un conjunto de requisitos de una lista priorizada llamada Product Backlog, de manera que las funciones desarrolladas al principio del proyecto son prioridad para el cliente. Al final de cada sprint se entrega un producto de software funcional en el ambiente requerido por el cliente.

\section{Propuesta: Technical Writer como parte del equipo Scrum}

Al respecto, presentamos la incorporación de un integrante más al Equipo Scrum, que lo llamaremos "Technical Writer", quien será el encargado de llevar toda la parte formal, es decir la documentación y construcción de artefactos desde las fases iniciales del 
proyecto hasta su culminación. El principal objetivo es la entrega de un sistema de gestión de calidad para proyectos de tamaño mediano y grande. En la Figura 1 se puede apreciar el nuevo rol dentro de los tres aspectos fundamentales de Scrum: Roles, artefactos y eventos.

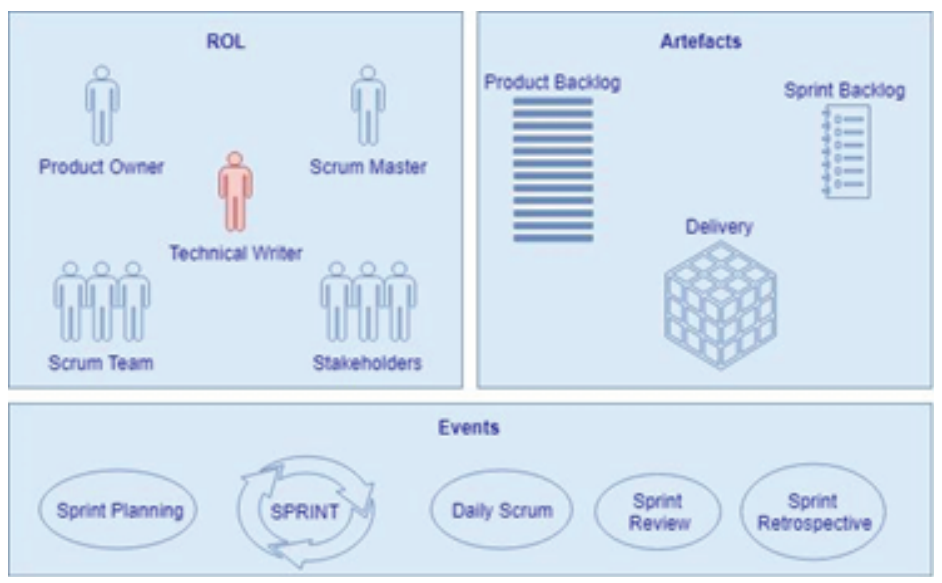

Figure 1: Componentes de Scrum.

\subsection{Componentes}

Para explicar mejor las funciones del "Technical Writer", explicaremos desde los tres aspectos antes mencionados:

\subsubsection{Roles}

Para nuestra propuesta, el Scrum Master sus responsabilidades no varían en absoluto, tendrá las mismas responsabilidades de analizar las necesidades tanto del Product Owner como las del equipo Scrum.

Para el caso de Product Owner se enfocará en la obtención de la información que será transmitida hacia el Scrum Master, dentro de los roles estará la planificación de los sprints y los desarrolladores en escribir las líneas de código (actividad relacionada a su formación).

Mientras que el Scrum Team se orienta a todas las personas que participan en el desarrollo del proyecto como son: programadores, diseñadores y los testers. Los Stakeholders se mantendrán con las personas que están interesadas en el producto software que se está desarrollando, pueden ser internos y externos.

El nuevo actor es: "Technical Writer" quien participará en todas las fases de Scrum [3], por ejemplo en la fase inicial llevará la visión general del producto, levantará las 
épicas, registrará el Backlog priorizado del producto. En la fase de Sprint Planning llevantará historias de usuario e identificará las tareas requeridas. En la fase de Sprint Review y Retrospective registrará el cumplimiento de las tareas ejecutadas, así como su versionamiento. Al término del proyecto se estará entregando junto con el producto software un documento final que consolida toda la documentación generada de cada sprint, cubriendo de esta forma la falencia de la metodología Scrum en cuanto a la documentación y entregando un valor agregado al cliente para satisfacer su necesidad, la misma que propone la norma ISO 9001:2015[11] en su apartado 7.5.

Product Owner y el Scrum Master, seguirán recopilando toda la información hasta transformarlo a un documento o artefacto formal siguiendo los estándares de la ISO 9001:2015 para el aseguramiento de un sistema de gestión de calidad, mismo que proporcionará al cliente un valor agregado.

El Technical Writer no interferirá en las tareas asignadas al equipo durante el proceso de cada sprint.

\subsubsection{Artefactos}

Los artefactos se mantienen los mismos que son parte de Scrum. Para el caso del Product Backlog contendrá toda la pila de requerimientos por parte del cliente. EI Sprint Backlog de la pila del backlog se priorizan los requerimientos que ingresaran a un Sprint, y el Delivery es el producto resultante que se entregará al cliente de forma continua.

\subsubsection{Eventos}

Para el caso de los eventos, los Sprint Planning tendrán lugar al principio de cada sprint. El número de sprints en una entrega sigue siendo definido por el equipo. Los Sprint seguirán apareciendo en cada uno de los ciclos que producen una entrega o incremento por lo general de 2 a 4 semanas. El Daily Scrum continua con la reunión diaria del equipo Scrum, el Sprint Review en la que el equipo presenta el incremento y el Sprint Retrospective que sirve para analizar y mejorar la forma de hacer Scrum.

En base a los componentes descritos, y siguiendo la metodología se tiene nuestra propuesta que se muestra en la figura 2. 


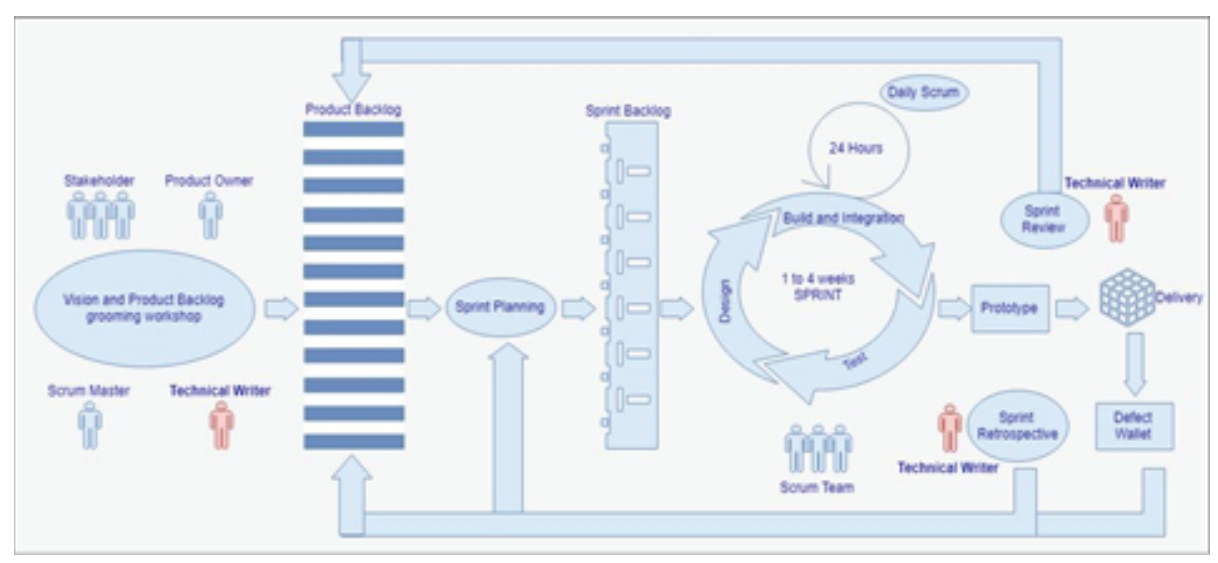

Figure 2: Propuesta la modelo de Scrum.

\section{Metodología y resultados iniciales de la propuesta}

En esta sección, mostramos la metodología y los resultados preliminares obtenidos al aplicar nuestra propuesta en una empresa de desarrollo de software en el Ecuador. En la cual, se llevó a cabo un estudio empírico. Para lo cual, se trabajó con dos equipos (equipo 1 y 2) de desarrollo Scrum, conformado por 4 integrantes cada equipo.

El equipo 1 se organizó de la siguiente manera: 3 desarrolladores, 1 ingeniero de calidad, mientras que el equipo 2 se organizó con 3 desarrolladores, 1 ingeniero de calidad a este equipo se le agregó un integrante más que es el Technical Writer. Para ambos equipos se designó a un solo Scrum Master y un Product Owner. Cada equipo planificó desarrollar el proyecto en 4 sprints, cada sprint con una duración de una semana.

El primer equipo desarrolló el proyecto de la manera tradicional utilizando la metodología Scrum, mientras que el segundo equipo aplicó nuestra propuesta, es decir, incluyeron a su equipo al nuevo actor llamado "Technical Writer".

La descripción del proyecto a desarrollar se muestra en la tabla 1.

TABLE 1: Descripción del Proyecto.

Proyecto
Tamaño del proyecto a desarrollar
Número de Iteraciones
Número de integrantes del equipo Scrum
Duración del proyecto
Características del Lenguaje de Programación
Lenguaje de Programación
Documentos
Ambiente de Desarrollo

Descripción
Pequeño-Mediano
4 semanas
4 miembros
1 mes
Orientado a Objetos
Java
Microsft Office
Netbeans IDE 8.2


En la reunión previa a este estudio empírico, se socializó a cada equipo los aspectos a evaluar, dando a conocer a cerca de la escala de valoración que es de 1 a 10, donde el valor de 1 es el menos significativo y 10 es el valor más significativo, esta escala en cuanto a la satisfacción o percepción de calidad tanto del equipo de desarrollo y el cliente.

Los valores obtenidos se muestran en la tabla 2.

TABLE 2: Datos del estudio empírico de ambos equipos Scrum.

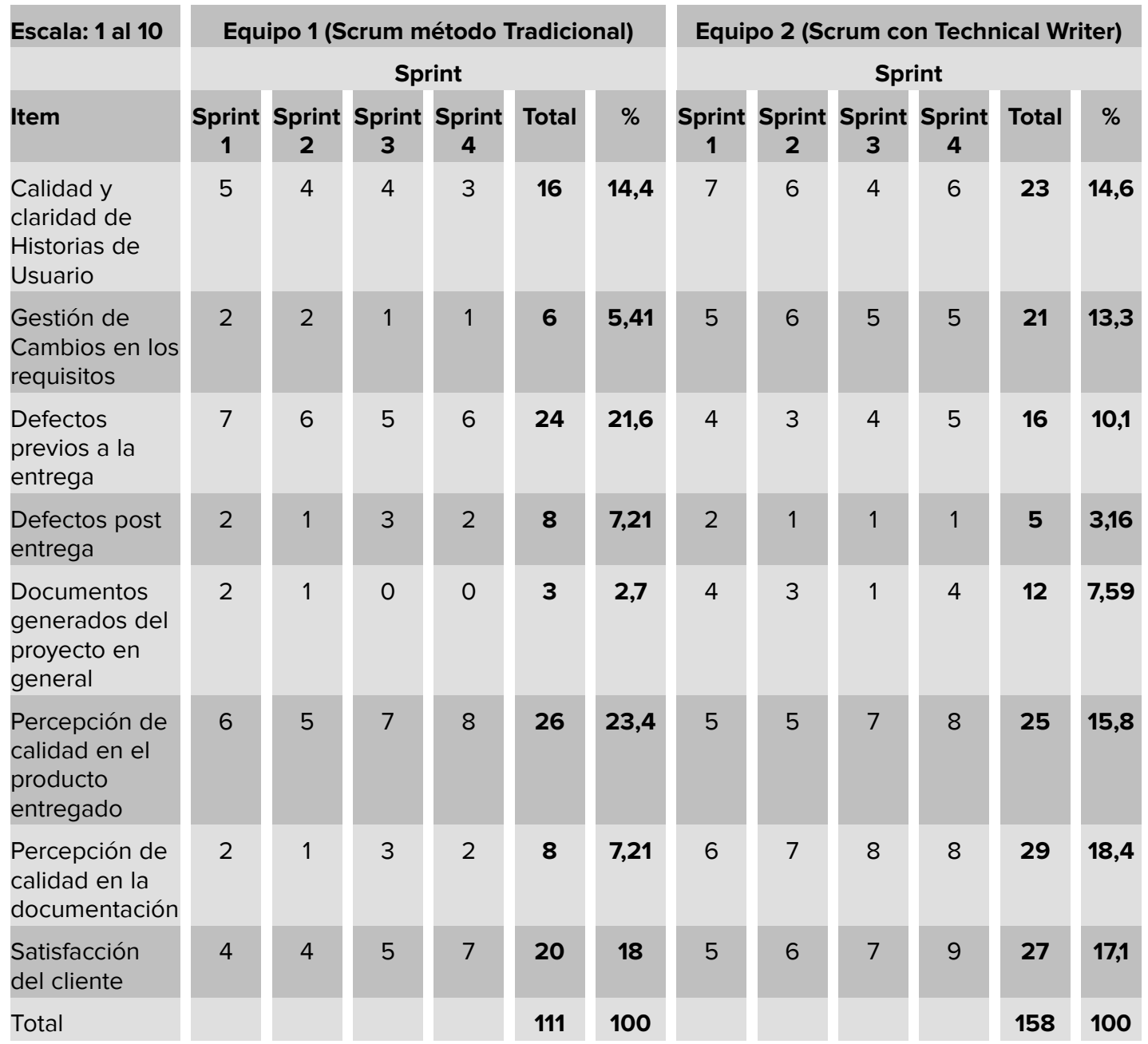

Los resultados obtenidos para nuestro estudio empírico demuestran que la gestión de la calidad del equipo 2 es mayor tanto del producto final así como en la documentación formal. Estos resultados fueron otorgados a la inclusión del nuevo integrante al equipo, mientras que el equipo 1 que sigue la metodología ágil Scrum (tradicional) es menor en cuanto a la gestión de la documentación entregada al cliente. 


\section{Conclusiones}

La participación del nuevo actor "Technical Writer" aportó significativamente al equipo 2 de Scrum con todo lo que a recolección de información se refiere, disponiendo a todo el equipo con la generación de toda la documentación del proyecto. La documentación realizada por el Technical Writer favoreció notablemente al equipo que desarrolló el software como antecedente en la norma ISO 9001:2015 en el camino a la implementación de documentación a todos los procesos involucrados en el proyecto de desarrollo con calidad.

El Technical Writer al tener funciones específicas de empoderamiento de los procesos documentales, apoya considerablemente al equipo de desarrollo, en temas de liberación de actividades de registro de evidencias documentales, fortaleciendo de esta manera el desarrollo continuo del proyecto y cumplimiento con los tiempos planificados de entregables para cada Sprint.

Según Christoph Stettina[1] (2011) citado en la sección 2, muestra los resultados de un estudio empírico relacionado a la documentación interna en equipos de desarrollo ágil (Scrum). Este considera que es muy limitada, incluso "insuficiente", por lo que creemos que el nuevo actor Technical Writer es necesario en el equipo para incorporar documentación apropiada para la transferencia de conocimiento y la mantenibilidad. Con nuestros resultados obtenidos sostenemos la hipótesis del autor y afirmamos nuestra propuesta que aportará a las empresas de desarrollo de software para afianzar sus procesos documentales y que permitirá disponer de toda la información necesaria para temas de mantenimiento, nuevos requerimientos, contingencias, y sobre todo como parte en el camino de la búsqueda de la certificación de todos sus proyectos basados en la norma ISO 9001:2015

\section{Trabajos futuros}

Implementar esta propuesta en otras empresas de desarrollo de software de tamaño mediano a grande que utilicen la metodología Scrum, en donde el proyecto amerite que se generen sprints de periodos más largos, y se pueda apreciar de mejor manera el impacto de tener un Technical Writer en el equipo. Finalmente, al basarse en la norma ISO 9001:2015 se dispondrá de información consolidad, bien documentada y de calidad. 


\section{References}

[1] Christoph Johann Stettina, Werner Heijstek. "Necessary and Neglected? An Empirical Study of Internal Documentation in Agile Software Development Teams" ACM 978-1-4503-0936-3/11/10, http://www.liacs.nl/home/\{stettina,heijstek\}

[2] Michael Coram and Shawn Bohner. " The Impact of Agile Methods on Software Project Management" doi: 0-7695-2308-0/05, Department of Computer Science, Virginia Polytechnical Institute and State University, \{mcoram, sbohner\}@vt.edu

[3] "SCRUM, it's about Common Sense., https://www.scrum.org/resources/what-isscrum

[4] Julieta Calabrese, Silvia Esponda, Marcos Boracchia, Patricia Pesado "Hacia una mejora de calidad en Scrum. Integrando documentación requerida por IRAMISO 9001:2015" Instituto de Investigación en Informática LIDI (III-LIDI), Facultad de Informática -- Universidad Nacional de La Plata, \{jcalabrese, sesponda, marcosb, ppesado\}@lidi.info.unlp.edu.ar

[5] A. Alliance. 2001 "Agile manifesto," [Online]. Available: http://agilemanifesto.org/ [Accessed 2505 2017]

[6] D. E. Strode, S. L. Huff, B. Hope, \& S. Link, "Coordination in co-located agile software development projects," Journal of Systems and Software, vol. 85, no. 6, pp. 1222-1238, 2012.

[7] J. Highsmith, and A. Cockburn, "Agile software development: The business of innovation," Computer, vol. 34, no. 9, pp.120-127, 2001.

[8] O. P. Timperi, "An overview of quality assurance practices in agile methodologies," In T-76.650 Seminar in Software Engineering, 2004.

[9] A. Cockburn, "Agile Software Development," Boston, MA, USA: Addison-Wesley Longman Publishing Co., Inc., 2002.

[10] P. Abrahamsson, J. Warsta, M. T. Siponen, and J. Ronkainen, "New directions on agile methods: A comparative analysis," In Proceedings of the 25th International Conference on Software Engineering, ser. ICSE '03. Washington, DC, USA: IEEE Computer Society, pp. 244--254, 2003.

[11] ISO. (2015). Sistemas de gestión de la calidad, Fundamentos y vocabulario. Suiza, Ginebra: International Organization for Standardization. 